\title{
Invasive Lobular Carcinoma Mimicking Papillary Carcinoma: A Report of Three Cases
}

\author{
Emad A. Rakha Areeg Abbas Rachael Sheeran \\ Nottingham University Hospitals NHS Trust, City Hospital Campus, Nottingham, UK
}

\section{Established Fact}

- Invasive lobular carcinoma can present with a growth pattern typical of solid papillary carcinoma. The lobular nature of these tumours can be confirmed by the absence of E-cadherin and $\beta$-catenin membrane expression.

\section{Novel Insight}

- Encapsulated and solid papillary carcinomas represent a unique growth pattern of breast carcinomas rather than reflecting the in situ or invasive nature of the tumour.

\section{Key Words}

Human breast carcinoma - Invasive lobular carcinoma . Encapsulated papillary carcinoma

\begin{abstract}
Encapsulated and solid papillary carcinomas (EPCs and SPCs) are considered historically as a special form of ductal carcinoma in situ. Invasive lobular carcinoma (ILC) is characterised by a discohesive growth pattern. There are several variants of ILC, but, as yet, no papillary subtype has been identified. Here we report 3 cases of ILC presenting as papillary carcinoma (PC) with a typical solid papillary growth pattern. One case was reported on core biopsy as EPC (B5a). The 3 ensuing resection specimens showed features typical of SPC
\end{abstract}

with a circumscribed malignant epithelial proliferation containing fibrovascular cores and surrounded, at least focally, by a thick fibrous capsule. The lobular nature of these tumours was confirmed on the resection specimens by the absence of E-cadherin and $\beta$-catenin membrane expression. The invasive nature was confirmed by the presence of entrapped fat cells, the absence of myoepithelial cells and focal merging of the solid papillary areas with classic ILC at the periphery. Of note, 1 case was a recurrent carcinoma without an in situ component. Conclusion: This study provides further evidence that EPC and SPC represent a unique growth pattern of breast carcinomas rather than reflecting the in situ or invasive nature of the tumour, and that ILC can acquire a papillary growth pattern.

(c) 2016 S. Karger AG, Basel

\section{KARGER}

E-Mail karger@karger.com www.karger.com/pat (c) 2016 S. Karger AG, Base

$1015-2008 / 16 / 0835-0221 \$ 39.50 / 0$
Dr. Emad A. Rakha

Division of Cancer and Stem Cells, Nottingham University Hospital NHS Trust City Hospital Campus, Hucknall Road

Nottingham NG5 1PB (UK)

E-Mail emadrakha@yahoo.com 


\section{Introduction}

Encapsulated and solid papillary carcinomas (EPCs and SPCs) of the breast are distinct histologic subtypes characterised by a well-defined, malignant, epithelial proliferation supported by fibrovascular stalks [1-4]. There is still controversy about the nature of papillary carcinoma (PC), but accumulating evidence indicates that PCs lacking myoepithelial cells have the biological potential to behave as an invasive disease with an expansile papillary growth pattern and indolent clinical behaviour. This is supported by the occasional development of lymphovascular invasion, lymph node metastases and recurrences in some cases with typical PC morphology [1-7]. PC has been reported to be of ductal origin, and, to our knowledge, all reported cases have been described as such.

Invasive lobular carcinoma (ILC), which represents 5-15\% of breast carcinomas, has several recognised growth patterns including classic, solid, alveolar, tubulolobular and pleomorphic [8]. In this report, we describe 3 cases of ILC with a PC growth pattern, providing further evidence that PC represents a unique growth pattern of breast carcinoma that does not always involve the expansion of the native breast ducts by malignant, papillary, ductal epithelial proliferation; this can be observed in ILC.

\section{Case Presentation}

No ethics approval or informed consent was required. The first case is of a woman who presented with a symptomatic lump in her right breast. There was no previous history of breast cancer. The tumour did not adhere to the skin and the axillary lymph nodes were not enlarged, either clinically or radiologically. Histological examination of the needle core biopsy (NCB) showed a well-defined, malignant, papillary lesion with cystic areas, a peripheral, capsule-like structure and focal hemosiderin deposition, suggesting previous intracystic bleeding. The proliferating atypical cells showed low-grade cytonuclear features. Immunohistochemistry (IHC) showed diffuse nuclear oestrogen receptor (ER) staining, negative HER2 expression and absent expression of the myoepithelial markers smooth-muscle myosin (SMM), smooth-muscle actin (SMA) and p63. The core lesion was diagnosed as NHSBSP core biopsy category B5a [malignant in situ/ductal carcinoma in situ (DCIS)] [9] of EPC type (fig. 1). Macroscopic examination of the excision specimen showed a $28-\mathrm{mm}$, well-defined, solid lesion. Histological assessment revealed a well-defined, focally encapsulated, solid mass of proliferating malignant cells featuring papillary architecture, with fibrovascular cores, similar to what was seen on the NCB (fig. 1, 2). However, there was focal extension of malignant cells from the PC-like mass into the surrounding tissue and these had a classic ILC growth pattern. Scattered small foci of classic ILC and lobular carcinoma in situ (LCIS) were also seen in the background breast tissue. Further IHC was thus performed which demonstrated an absence of membrane expression of E-cadherin and $\beta$-catenin in the papillary and classic ILC areas, confirming the lobular nature of the lesion. Myoepithelial markers (SMM, SMA and p63) and neuroendocrine markers (synaptophysin and chromogranin A) were negative. A diagnosis of ILC with a PC growth pattern was made. Supporting fibrovascular cores typical of EPC and SPC were apparent using HE staining and on IHC (SMA). These, in addition to the presence of a well-defined margin with a focal peripheral fibrous tissue capsule/ pseudocapsule, differentiated it from the solid variant of ILC. No lymphovascular invasion was identified and the sentinel lymph node was negative $(0 / 1)$.

The second case is of a woman who presented with a suspicious mass in her right breast. There was a history of ipsilateral ILC 16 years previously and recurrent disease was suspected. The NCB showed an ER-positive, HER2-negative carcinoma with a solid growth pattern that was reported as ductal carcinoma no special type (duct/NST) with lobular features. No E-cadherin staining was carried out. The mastectomy specimen contained multiple, wellcircumscribed masses, ranging in size from 3 to $7 \mathrm{~mm}$, with typical PC features associated with classic ILC extending over an area 22 $\mathrm{mm}$ in size. On IHC, there was no membrane expression of Ecadherin or $\beta$-catenin and the absence of myoepithelial cells within the PC mass lesions was confirmed. This tumour was diagnosed as grade 2 ILC, consistent with a recurrence of the previously diagnosed ILC. No lymphovascular invasion or in situ component was identified. Sentinel lymph nodes were negative (0/3). No evidence of intraductal papillary lesion was identified in the current or previous specimens.

The third case was a woman whose NCB revealed a classic ILC. The resection specimen showed a 6-mm focus of typical PC associated with classic ILC, extending over an area $23 \mathrm{~mm}$ in size. IHC showed negative membrane expression of E-cadherin and $\beta$-catenin, and confirmed the absence of myoepithelial cells within the PC focus (fig. 3). The tumour was grade 2, no lymphovascular invasion or in situ carcinoma was identified and the lymph nodes were negative $(0 / 3)$.

No significant events were identified within a median followup time of 13 months.

\section{Discussion}

PC of the breast has long been considered as a special variant of DCIS with an expansile growth pattern. There is compelling evidence to support the potential invasive nature of PC $[2,4-6,10]$, but the molecular mechanisms underlying this unique growth pattern and the ultimate behaviour of these lesions remains to be defined. The current recommendation is to manage PC as an in situ disease similar to DCIS (carcinoma in situ) [4]. Although most authors $[5,11]$ consider EPC synonymous with intracystic (encysted) PC but not SPC and these were described separately in the latest (4th) edition of the 'WHO 

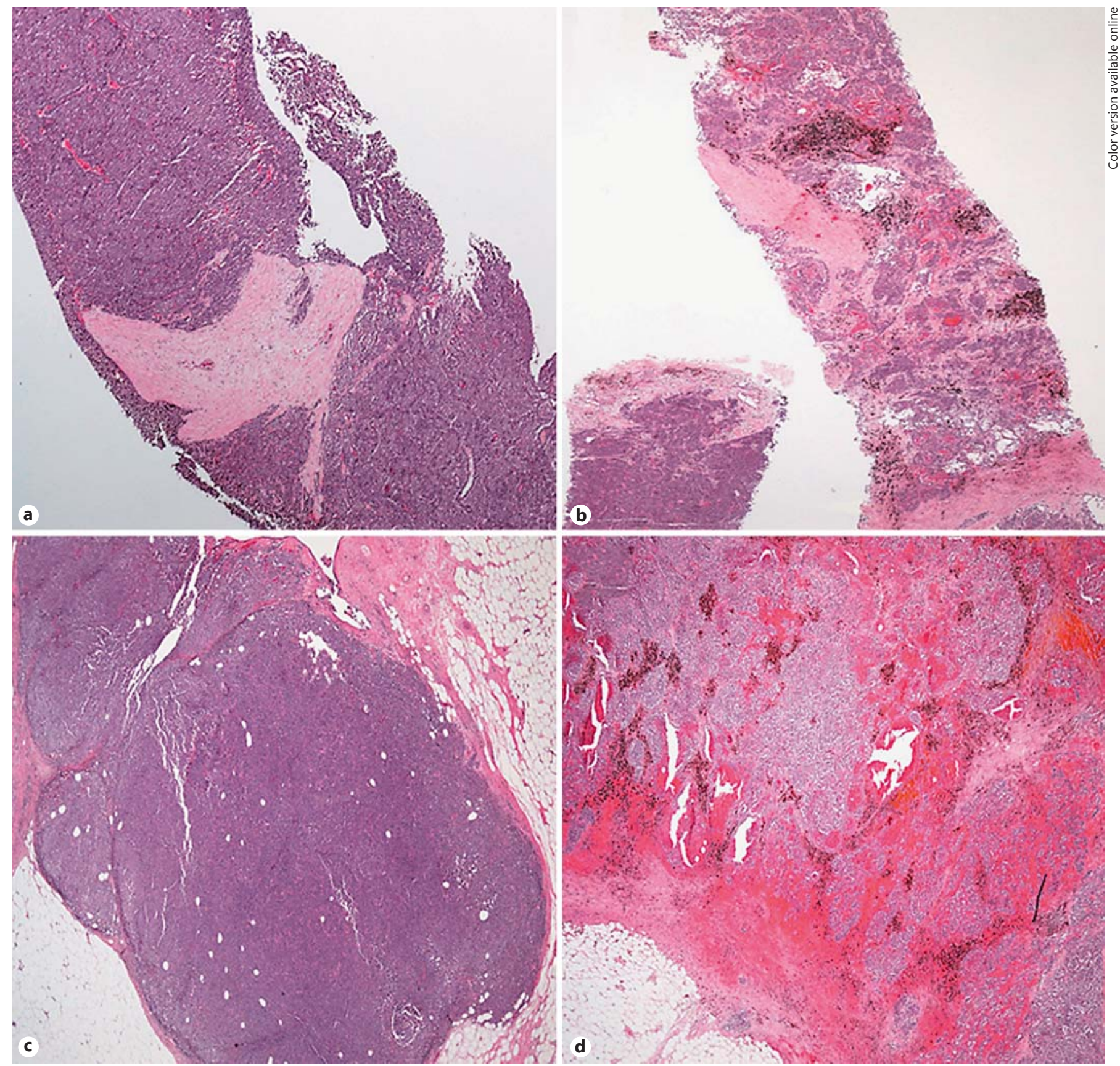

Fig. 1. a NCB of the first case, showing features typical of EPC with a solid papillary malignant tumour and thick fibrous connective tissue. $\mathbf{b}$ NCB from the same tumour, showing hemosiderin deposition in keeping with previous intracystic haemorrhage. $\mathbf{c}$ Resection specimen of the same case, showing EPC with features similar to those seen in $\mathbf{a}$ and featuring entrapped fat cells. $\mathbf{d}$ Resection specimen, showing areas of hemosiderin deposition similar to those seen in $\mathbf{b}$.

Classification of the Tumours of the Breast' [4], some cases show overlapping features [2]. EPCs may show solid areas and some SPCs show peripheral encapsulation and lack neuroendocrine differentiation. In addition, the pap- illary cores characteristic of PC vary widely from welldeveloped cores in EPC to rudimentary papillary stalks in SPC that may require IHC to demonstrate the presence of fibrovascular cores within the lesion [12]. Moreover, 

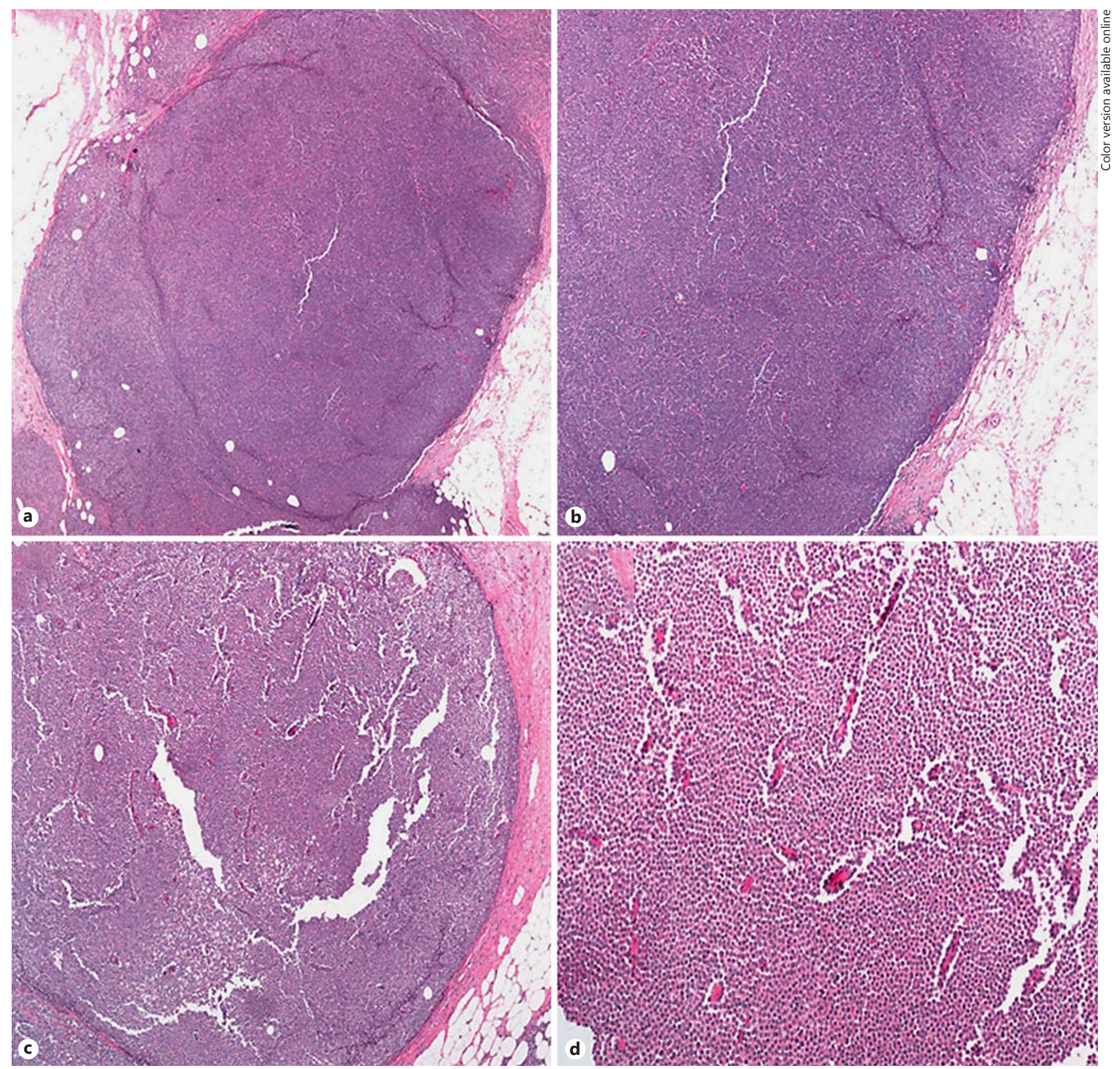

Fig. 2. a, b Different pictures of the first case, featuring a well-defined margin and peripheral fibrous tissue. c, $\mathbf{d}$ The second case, with well-developed fibrovascular cores typical of papillary lesions.

although solid ILC is a recognised variant of ILC, in this specific subtype, the cells with lobular morphology grow in sheets, lack papillary cores and peripheral encapsulation and are typically more pleomorphic with a high frequency of mitotic figures [8].
In this study, we present 3 cases of ILC which formed a well-defined focally encapsulated malignant tumour mass, each containing fibrovascular cores characteristic of PC. In the first case, the PC was the predominant growth pattern. The papillary mass was sampled on the 

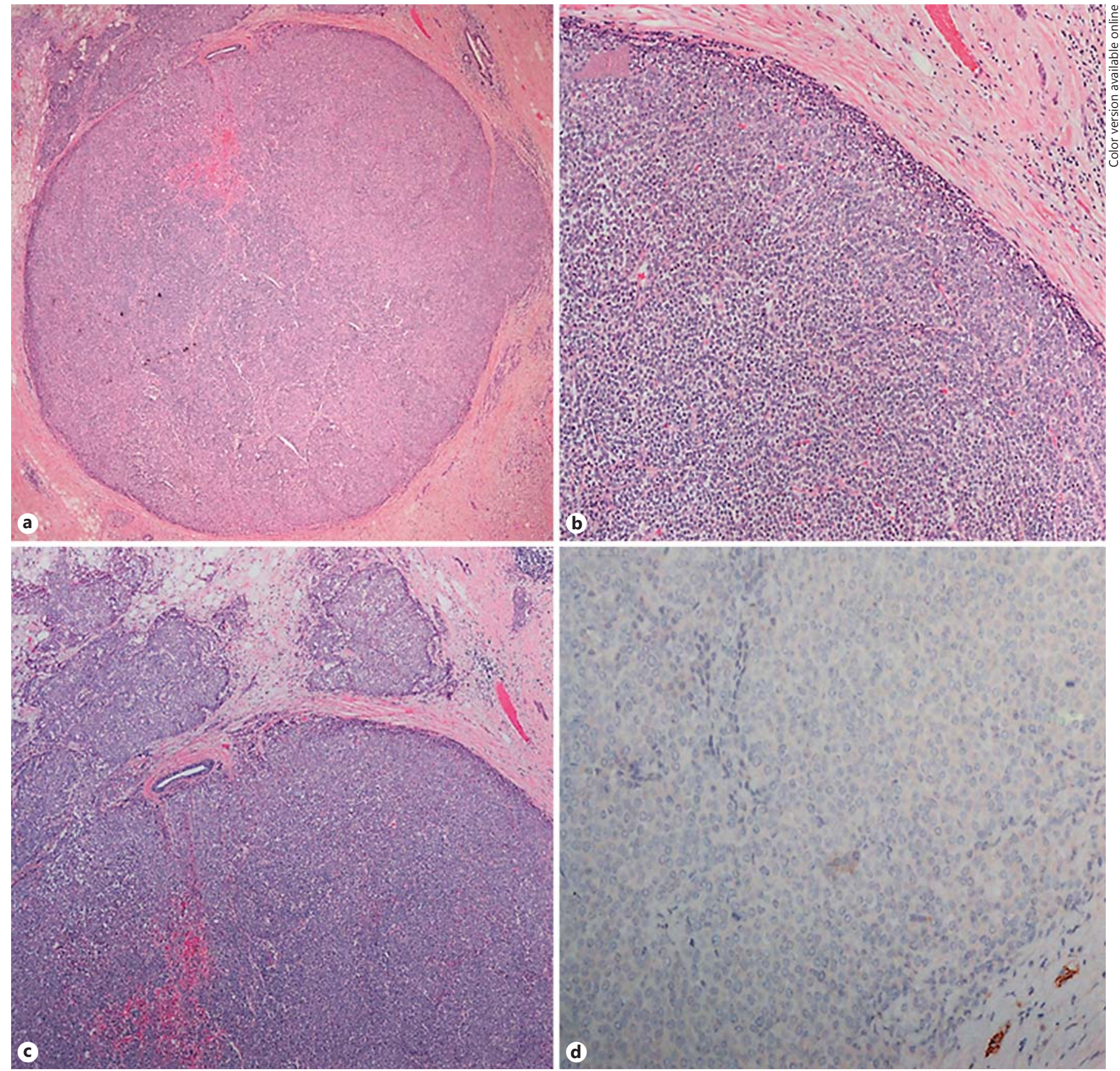

Fig. 3. The third case, featuring a circumscribed papillary nodule (a) with a focal, thick fibrous capsule (b). c Focal extension of the tumour, showing papillary architecture outside the capsule. $\mathbf{d}$ Negative E-cadherin expression.

NCB and was therefore diagnosed as EPC (B5a [9]). ILC was not suspected and IHC to confirm lobular subtype was not carried out. This mainly stemmed from the following: (1) the discohesion of malignant cells was not obvious, (2) lobular carcinomas-like PC may show plasma- cytoid appearance and may exhibit strong diffuse nuclear ER positivity and (3) there is a lack of reported lobular neoplasia in papillary lesions. In the other 2 cases, a diagnosis of duct/NST with lobular features and ILC was rendered, respectively. 
In all 3 of our cases, the lobular nature of the tumour was confirmed in the resection specimen by IHC, which demonstrated the absence of E-cadherin and $\beta$-catenin membrane expression. Although it has been reported that ductal carcinomas may show an aberrant loss of expression of E-cadherin and $\beta$-catenin, this is typically focal and observed in high-grade tumours [13]. The similarity of the cytonuclear morphology and immunoprofile between PC-like areas and classic ILC areas present in the surrounding breast tissue provided further evidence to support the lobular nature of the lesions. However, there is the possibility that the 'PC-like' mass lesions were the result of the expansion of multiple adjacent foci of LCIS or LCIS involving an intraductal papilloma. No previous history of intraductal papillary lesions was identified in these patients. The invasive nature of PC-like masses was confirmed by the absence of myoepithelial cells within and at the periphery of the EPC, the profound lack of residual ductal cells within these lesions, focal merging of the 'PC-like' areas with the more classic ILC growth pattern and the presence of entrapped fat cells among the proliferating malignant cells (fig. 1c).

One of these cases represented a recurrent carcinoma. The demonstration of fibrovascular cores so characteristic of PCs within the 'PC-like' mass lesions could argue against the possibility of an adjacent expansile mass of the solid or alveolar subtypes of ILC, which would thus mimic PC. This was also supported by the presence of the focal extension of tumour cells outside the PC-like masses with the preservation of a papillary growth pattern (fig. 3c). A well-defined, solid growth pattern containing fibrovascular cores of variable thickness is a characteristic feature of SPC of the breast and its papillary nature may need to be demonstrated using IHC [14].

To our knowledge, this is the first report of ILC demonstrating a PC pattern. There is an earlier case report which includes a biphasic ILC and a papillary breast carcinoma (collision tumour) [15]. Our study provides further evidence that PC represents a unique growth pattern of breast carcinomas that does not always denote the in situ or ductal nature of the disease. It is important to document that ILC presenting as PC is similar to other variants of ILC on IHC; these lesions also show ER-positive and HER2-negative phenotypes and lack E-cadherin and $\beta$-catenin membrane staining. Although we support the current consensus recommendation that low- and intermediate-grade PCs of ductal type should be managed as an in situ disease (carcinoma in situ) based on their indolent behaviour that does not justify the use of systemic therapy, this study provides evidence that ILC can present as PC. ILC with PC growth pattern can be included in the differential diagnosis of PC on core biopsy. Sentinel lymph node biopsy and consideration for systemic therapy based on agreed guidelines for the management of invasive breast cancer should be considered if PC-like tumour features a lobular phenotype. Further studies on recognising and investigating lobular PC will most likely provide further understanding of the biology and behaviour of these tumours.

\section{Disclosure Statement}

There were no conflicts of interest.

\section{References}

1 Rosen PP: Rosen's Breast Pathology. Philadelphia, Lippincott Williams \& Wilkins, 2009.

2 Rakha EA, Gandhi N, Climent F, van Deurzen $\mathrm{CH}$, Haider SA, et al: Encapsulated papillary carcinoma of the breast: an invasive tumor with excellent prognosis. Am J Surg Pathol 2011;35:1093-1103.

3 Pal SK, Lau SK, Kruper L, Nwoye U, Garberoglio C, et al: Papillary carcinoma of the breast: an overview. Breast Cancer Res Treat 2010;122:637-645.
4 Collins L, O’Malley F, Visscher D, Moriya T, Ichihara S, Reis-Filho JS: Encapsulated Papillary Carcinoma; in Lakhani SR, Ellis IO, Schnitt SJ, Tan PH, van de Vijver MJ (eds): WHO Classification of Tumours of the Breast, ed 4. Lyon, IARC Press, 2012, pp 106-109.

5 Esposito NN, Dabbs DJ, Bhargava R: Are encapsulated papillary carcinomas of the breast in situ or invasive? A basement membrane study of 27 cases. Am J Clin Pathol 2009;131: 228-242.

6 Rakha EA, Tun M, Junainah E, Ellis IO, Green A: Encapsulated papillary carcinoma of the breast: a study of invasion associated markers. J Clin Pathol 2012;65:710-714.
7 Rakha EA, Varga Z, Elsheik S, Ellis IO: Highgrade encapsulated papillary carcinoma of the breast: an under-recognized entity. Histopathology 2015;66:740-746.

8 Lakhani SR, Rakha EA, Simpson PT: Invasive Lobular Carcinoma; in Lakhani SR, Ellis IO, Schnitt SJ, Tan PH, van de Vijver MJ (eds): WHO Classification of Tumours of the Breast, ed 4. Lyon, IARC Press, 2012, pp 140142.

9 NHSBSP Breast Screening Programme: Guidelines for Non-Operative Diagnostic Procedures and Reporting in Breast Cancer Screening. Sheffield, NHSBSP Publication No. 50, June 2001. 
10 Duprez R, Wilkerson PM, Lacroix-Triki M, Lambros MB, Mackay A, et al: Immunophenotypic and genomic characterization of papillary carcinomas of the breast. J Pathol 2012; 226:427-441.

11 Collins LC, Schnitt SJ: Papillary lesions of the breast: selected diagnostic and management issues. Histopathology 2008;52:20-29.
12 Rakha EA: Morphogenesis of the papillary lesions of the breast: phenotypic observation. J Clin Pathol 2016;69:64-69.

13 Rakha EA, Teoh TK, Lee AH, Nolan CC, Ellis IO, Green AR: Further evidence that E-cadherin is not a tumour suppressor gene in invasive ductal carcinoma of the breast: an immunohistochemical study. Histopathology 2013;62:695-701.
14 Tse GM, Tan PH, Moriya T: The role of immunohistochemistry in the differential diagnosis of papillary lesions of the breast. J Clin Pathol 2009;62:407-413.

15 Ang D, VanSandt AM, Beadling C, Warrick A, West RB, et al: Biphasic papillary and lobular breast carcinoma with PIK3CA and IDH1 mutations. Diagn Mol Pathol 2012;21:221224 